\title{
Aperçus sur la similitude des modèles réduits destinés à l'étude des seiches portuaires
}

\section{Note on the similitude of scale models for studying seiche in harbours}

\author{
PAR F. BIESEL ET B. LE MEHAUTÉ \\ INGÉNIEURE AU LABORATOHE DALPHINOIS D'HYOTAUJIQUE; \\ (SOGREAH, GRENOBIE)
}

\begin{abstract}
Pont limiter l'amplitude et In fiequence des seiches dans les ports, on pent songer a absorber (ou d évacuer) l'énergic incidenic. Les dispositi/s préconisés sont, en général, efficaces pouir toutes les périodes, mais demandent sontpent des travaux de génie civil importants.

II est parfois possible, non pas de supprimer tes résonances dans les clarses du port, mais de s'assurer qu'elles correspondent à des périodes pour lesquelles l'excitation se présente avec une amplitude très faible.

Fn effet, alors que les houles de tempete ont, au voisinage des ports, une anplitnde relativement indépendante de la période, il n'en est pas toujours de même des ondes génératrices de seiches. Ces ondes, par suite de leur réfraction sur les plus grands fonds marins, de leur réflexion sur les côtes les plus plates, subissent des phénomènes de convergence et provoquent des phénomènes de résonance dans les baies ou sur le platean continental avoisinant le port. Pour certaines périodes, l'amplitude peut être parficulièrement amplifiée el la répartition spectrale des ondes génératrices de seiches très irrégulière.

Il est donc parfois diffeilement pensable d'étudier les seiches dans un port sans lier celui-ci aux phénomènes qui l'entourent et qui sont inconnus dans la majorifé des cas. L'étude du port isolé peut donner les périodes de résonance, mais ne donnera pas les amplitudes relalives en fonction de la période.

La technique du modèle réduit doit donc être pensée d̀ l'échelle du phénomene, c'est-du-dire à l'échelle océanique. Les conditions aux limites doivent satisfaire à des impératifs spécifiques entrainant une conception dil modele différente de celle des modèles à houle.
\end{abstract}

In order to limit the amplitude and frequency of seiches in harbours, it is possible to envisage absorbing (or evacuating) the incident energy. The denices for this purpose are generally effective for all periods, but often require large scale construction work.

It is sometimes possible, rather than suppressing the resonances in the harbonr basins, to ensure that they correspond to periods for which the excitation occurs with a very small amplitude.

In reality, while storm wanes have, when in the neighbourhood of the port, an amplilute relatively independent of the period, this is not always so for the wanes that generate seiche. These waves, after being refracted in the greatest ocean depths and reflected from the flattest coastlines, undergo convergence phenoment and cause resonance phenomena in bays or on the continental shelf neighbouring the port. For certain periods the amplitude can be very much amplified and the spectral distribution of the waves causing the seiche very irregular.

Consequently it is sometimes difficult to consider a seiche study for a port, without relating it to the phenomena in the surrounding area, which are in most cases unknown. The study of the isolated port can give resonance periods but will not give the relative amplitudes as a function of the period.

The technique for the scale model must therefore be thought of on the same scale as the. phenomena, i.e. on an oceanic scale. The boundary conditions must satisfy specific conditions which involve a different design of the model from that for ordinary wave models.
L'étude des seiches portuaires est relative. ment nouvelle dans la technique du modèle réduit et pose de nombreux problèmes de similitude [1]. Nous ne cherchons pas ici à être complets et à faire le tour de toutes les questions qui se posent; nous ne cherchons pas plus à donner un compte rendu détaillé des études que le Labo- ratoire Dauphinois d'Hydraulique a en cours sur le sujet. Notre but est uniquement de faire le point des conclusions que nous considérons à l'heure actuelle comme à peu près acquises et d'énoncer les principales règles de similitude qui en découlent. Nous espérons provoquer ainsi des échanges de vue aussi larges que possible. 


\section{I. - RAPPEL SUCCINCT DES NOTIONS GÉNÉRALES SUR LA SIMILITUDE}

La « meilleure » échelle n'est pas la plus grande possible, contrairement à une opinion trop rẻpandue. En fait, les échelles des modèles réduits doivent se déterminer essentiellement par des considérations économiques.

Il est clair par exemple qu'un modèle qui coûte 10 millions et permet d'escompter une économie de l'ordre de 200 millions est préférable à un modèle plus grand et plus précis, permettant peut-être d'économiser 20 millions de plus mais coûtant 50 millions au lieu de 10 .

En fait, les éléments de la décision se présentent rarement d'une façon aussi précise. Il est en général presque impossible d'évaluer, même grossièrement, " l'espérance d'économie 》 d'un modèle et surtout la variation de cette économie en fonction de la précision des essais. La détermination des échelles de réduction optimum sera donc nécessairement plutôt un art qu'une science, mais cependant la valeur des résultats obtenus dépendra avant tout de la somme de connaissances dont pourra disposer le projeteur. Parmi ces connaissances, les lois de similitude sont primordiales car, non seulement elles permettront d'estimer le degré de précision à attendre d'un modèle, mais surtout elles permettront une conception saine de ce dernier et l'obtention de la précision optimum compatible avec un budget de recherche déterminé.

La première étape de l'étude de la similitude n'est pas la discussion des équations de détail mais essentiellement un examen d'ensemble du problème et de sa nature physique. Il est impossible, par exemple, de concevoir sainement un modèle sans avoir une idée à priori sur les causes et origines du phénomène étudié. C'est pourquoi, pour pouvoir discuter de la conception de modèles, nous serons amenés à faire une hypothèse sur l'origine des seiches, quoiqu'il n'entre pas dans nos intentions de discuter ici le problème qui fait l'objet d'un chapitre spécial dans l'étude dont cet exposé est tiré.
L'examen d'ensemble des phénomènes en jeu permet d'éliminer à priori les façons foncièrement défectueuses d'aborder le problème; il permet de tracer à grandes lignes les frontières du raisonnable. Très souvent il permettra aussi de déterminer les principales étapes de l'étude et éventuellement le nombre et la nature des modèles nécessaires.

L'étude de détail des équations des phénomènes viendra ensuite préciser les approximations, les échelles, les distorsions, etc., et définitivement le nombre des modèles nécessaires et les règles de similitude applicables à chacun d'eux.

L'allusion que nous venons de faire à la nécessité éventuelle de construire plusieurs modèles ne surprendra pas les spécialistes qui savent bien que cela est souvent plus économique que de vouloir tout étudier sur un modèle unique. Pour une banale étude de protection d'un port contre la houle, il peut arriver que l'on étudie l'ensemble des installations au $1 / 150$, quelques ouvrages particulièrement importants (ouvrages d'entrée par exemple) au $1 / 75$ et la stabilité des ouvrages individuels au $1 / 40$.

Nous allons voir que pour l'étude des seiches il pourra être nécessaire de faire un modèle à une échelle de l'ordre du $1 / 1.000$ et que sí l'on désire ćtudier aussi bien les seiches de 4.0 secondes que les seiches de 4 minutes, il pourra même être nécessaire de faire deux modèles différents, quoiqu'il s'agisse de l'étude d'un même phénomène, seul un paramètre ayant changé. Heureusement l'étude du mouvement des navires amarrés permet en général de réduire la gamme des périodes à étudier et ainsi d'éviter les modèles multiples.

En résumé, le problème de la similitude est loin d'être simple et de s'accommoder de solutions standardisées. L'ingénieur dispose d'un ensemble de moyens complexes dont il uoit savoir jouer avec habileté. Mieux il connaîtra les outils à sa disposition, plus les solutions seront efficaces et économiques.

\section{II. - HYPOTHESE SUR L'ORIGINE DES SEICHES}

Ainsi que nous l'avons mentionné plus haut, il n'est pas possible de concevoir un modèle sans admettre au moins quelques hypothèses sur l'origine des phénomènes à étudier. Il est clair qu'un modèle d'étude de seiches portuaires sera organisé de façon complètement différente selon que l'on supposera que les seiches proviennent du large sous forme d'onde progressive ou sont dues par exemple à des variations locales de pression atmosphérique.

Dans le but de limiter notre exposé et de le rendre plus clair, et non dans le but de prendre parti sur l'origine des seiches, nous étudierons un modèle conçu en fonction de la première hy- 
pothèse mentionnée ci-dessus, c'est-à-dire que nous admettrons que les mouvements de seiches sont provoqués par ces ondes progressives ou « ondes de seiche » venant du large.

Cette hypothèse présente, pour notre propos, les avantages suivants :

- Elle est plausible et assez généralement acceptée;

- Elle permet de discuter la plupart des problèmes de similitude qui se posent pour les modèles construits en fonction d'autres hypothèses;

- Elle permet une comparaison facile et ins- tructive avec l'étude des houles de tempêtes;

- Elle conduit à des modèles relativement faciles à exploiter (d'ailleurs la majorité des études de seiches sur modèles réduits est basée, au moins implicitement, sur cette hypothèse).

En première analyse, les études de seiches se présentent, dans cette hypothèse, de la même façon que les études de houle. Cette analogie eșt utile mais dangereuse si on l'applique sans discernement. C'est pourquoi l'essentiel de ce qui va suivre est une liste des différences existant entre l'étude des seiches et celle des houles.

\section{III. - DIFFERENCES ESSENTIELLES ENTRE LES HOULES DE TEMPETE ET LES ONDES DE SEICHE}

Les ondes de seiche font, ainsi que les vagues de tempête, partie de la grande famille des ondes de gravité. Celles-ci ne diffèrent de celleslà que par l'ordre de grandeur de certains paramètres, en particulier :

LA PÉRIODE. - L'ordre de grandeur des périodes les plus gênantes pour les navires en ce qui concerne les seiches est de 2 minutes, tandis qu'une période de 12 secondes, soit dix fois moins, est déjà assez longue pour la houle.

La cambrure. - Les houles de tempête dangereuses ont au large des cambrures de quelques pour cent n'approchant que très exceptionnellement la cambrure limite théorique de $14 \%$.

Au contraire, les ondes de seiches ont au large des cambrures beaucoup plus faibles. Par exemple une onde de $2 \mathrm{mn}$ a par grands fonds (plusieurs milliers de mètres) une longueur de l'ordre de $20 \mathrm{~km}$, tandis que son amplitude peut être de l'ordre de $2 \mathrm{~cm}$ seulement. Ce dernier chiffre est raisonnable car une telle onde aurait environ $20 \mathrm{~cm}$ d'amplitude en arrivant sur des fonds de 10 mètres (dans le cas d'une propagation bi-dimensionnelle, sans réflexion). En conséquence, la cambrure au large des ondes de seiches est de l'ordre de $10^{-6}$ (1 millionième), soit $\mathbf{1 0 . 0 0 0}$ fois plus petite que celle des houles de tempêtes les plus allongées.

Avant de tirer de ces faits les conclusions applicables à la technique des modèles réduits, il est bon, croyons-nous, de s'attarder à méditer sur leur signification. Il faut que l'esprit, habitué aux problèmes portuaires usuels, acquière une nouvelle optique pour l'étude des phénomènes de seiches. Il faut s'habituer à penser ces phénomènes dans leur cadre naturel et dans toute leur étendue.

La différence d'ordre des grandeurs des périodes, de 1 à 10 par exemple, se traduit par une disproportion encore plus grande des régions à considérer dans l'étude. On sait que le rapport des périodes étant de 10, le rapport des longueurs d'ondes, à profondeur relative égale, est de 100. Ceci change complètement l'échelle des phénomènes: là où pour les houles il fallait considérer un rayon d'une dizaine de kilomètres par exemple autour du port, il faut pour les seiches considérer, en première analyse, un rayon de $1.000 \mathrm{~km}$. Là où l'on se préoccupait de l'influence des fonds jusqu'à quelques 50 ou $100 \mathrm{~m}$, il faut maintenant aller jusqu'à des fonds de 5.000 ou $10.000 \mathrm{~m}$. Autrement dit, il faut se placer dans le cadre d'un océan entier, et nous verrons plus loin que même un tel cadre peut être trop étriquẹ́.

Prenons un exemple : en profondeur infinie, une onde de $2 \mathrm{mn}$ aurait une longueur d'environ $22,5 \mathrm{~km}$. Les fonds commencent donc à avoir une influence sensible sur sa propagation pour des profondeurs inférieures à environ $10.000 \mathrm{~m}$. Autrement dit, de telles ondes ne sont, pour ainsi dire, nulle part en profondeur \& pratiquement infinie »; elles subissent des réfractions même en plein océan et ne se propagent qu'exceptionnellement en ligne droite.

En supposant connue l'origine des ondes de seiches (par exemple une zone de perturbation atmosphérique) on ne peut étudier leur propagation que par des épures de réfractions, analogues aux « plans de vagues » mais couvrant parfois la presque totalité d'un océan. Ces épures pourront révéler des, effets de «lentille » con- 
centrant les ondes de seiches sur certains points des côtes et ainsi expliquer pourquoi certains ports sont particulièrement sujets à ce genre de phénomène.

On sait que les houles ont une longévité extraordinaire et que l'on a pu suivre leur propagation pendant plusieurs jours sur des milliers de kilomètres. Ơn sait également, à la suite d'études théoriques et d'observations, que plus les. ondes sont longues plus leur longévité est grande. On ne connaît pas exactement la loi qui lie le taux d'amortissement à la longueur d'onde, ou à la période, mais si l'on admettait que les distances parcourues sont, à amortissement égal, proportionnelles aux longueurs d'onde, on en concluerait que les ondes de seiches pourraient parcourir des centaines de milliers de kilomètres. c'est-à-dire faire de nombreuses fois le tour de la terre, avant d'être amorties.

L'extraordinaire petitesse de la cambrure des seiches vient encore renforcer leur longévité, sans doute en réduisant l'effet relatif de la turbulence, mais surtout en permettant la réflexion des ondes de seiche sur des côtes où des houles ordinaires seraient pratiquement entièrement amorties.

Nous abordons là un aspect extrêmement important du comportement des ondes de seiches.

Des études expérimentales ont confirmé le fait, prévu par la théorie, que plus la cambrure d'une houle était petite, mieux elle se réfléchissait sur une plage de pente donnée [4]. Les essais n'ont pas pu porter, évidemment, sur des houles ayant une cambrure de $10^{-6}$ en eau profonde, mais on dispose de formules théoriques permettant des extrapolations raisonnables. On sait en particulier que M. Mrche a montré qu'il y avait théoriquement réflexion totale si on avait :

$$
\gamma<\sqrt{\frac{2 \alpha}{\pi}} \cdot \frac{\sin ^{2} \alpha}{\pi}
$$

Soit, pour des plages très douces:

$$
\gamma<0,2541 . \alpha^{5 / 2}
$$

$\gamma$ étant la cambrure $2 a / L$ et $\propto$ la pente de la plage [9].

On en déduit que les ondes de cambrures $10^{-6}$ se réfléchissent parfaitement sur toutes les plages lisses de pente supérieure à $0,83 \%$. La plupart des plages seraient donc aussi réfléchissantes que des murs verticaux à l'égard des seiches. Par un raisonnement différent, M. Iribarren est arrivé à des conclusions analogues.

On voit que les ondes de seiches une fois créées, sont particulièrement durables. Elles s'usent extrêmement peu en se propageant et, de plus, peuvent se réfléchir sans perte notable d'énergie sur les côtes les plus fatales aux vagues ordinaires.
Il est probable, par exemple, que la longue plage rectiligne des Landes constitue un magnifique miroir qui renvoie les ondes de seiches provenant de l'Atlantique Nord ver's les côtes basques espagnoles. Ces ondes réfléchies viennent renforcer l'action des ondes directes incidentes. On sait d'ailleurs, depuis les remarquables travaux du professeur Iribarken, que de nombreux ports de cette côte sont gênés par des phénomènes de seiches [5] (fig. 1).

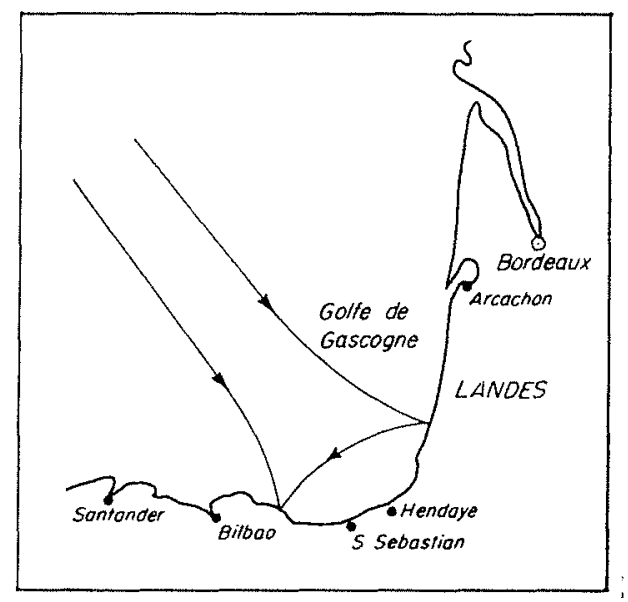

Fig. 1. - La longue plage des Landes constitue un magni* fique miroir qui renvoie les ondes de sciches vers les côtes basques espagnoles. Ces ondes viennent renforecr l'action directe des ondes incidentes.

Les côtes découpées sont probablement de moins bons réflecteurs et il est plus hasardeux, quoique tentant, de dire que la côte française entre la Bretagne et Biarritz participe au renforcement des seiches dans les ports cantabriques à la façon d'un gigantesque miroir concave.

Le port du Cap est un exemple possible de " miroir concave » (fig. 2). Les ondes de seiches, après diffraction autour de Robben Island, viennent se réfléchir sur la côte de la baie de la Table. L'énergie réfléchie se concentre à l'entrée des bassins et l'agitation est encore amplifiée par la présence de la jetée située au nord du bassin Victoria.

La baie d'Alger se présente, elle aussi, comme un « miroir concave » pour les ondes de seiches (fig. 3). La comparaison entre les figures 2 et 3 permet d'apprécier la grande influence de la présence d'une île comme Robben Island sur la propagation des ondes génératrices de seiches.

Nous arrêtons là cette discussion, susceptible, on le conçoit, de développements considérables. La longévité des ondes de seiche et la facilité avec laquelle elles se réfléchissent justifient en théorie de se pencher sur une mappemonde pour y suivre leurs cheminements à travers les océans et leurs rebondissements de continent en conti- 


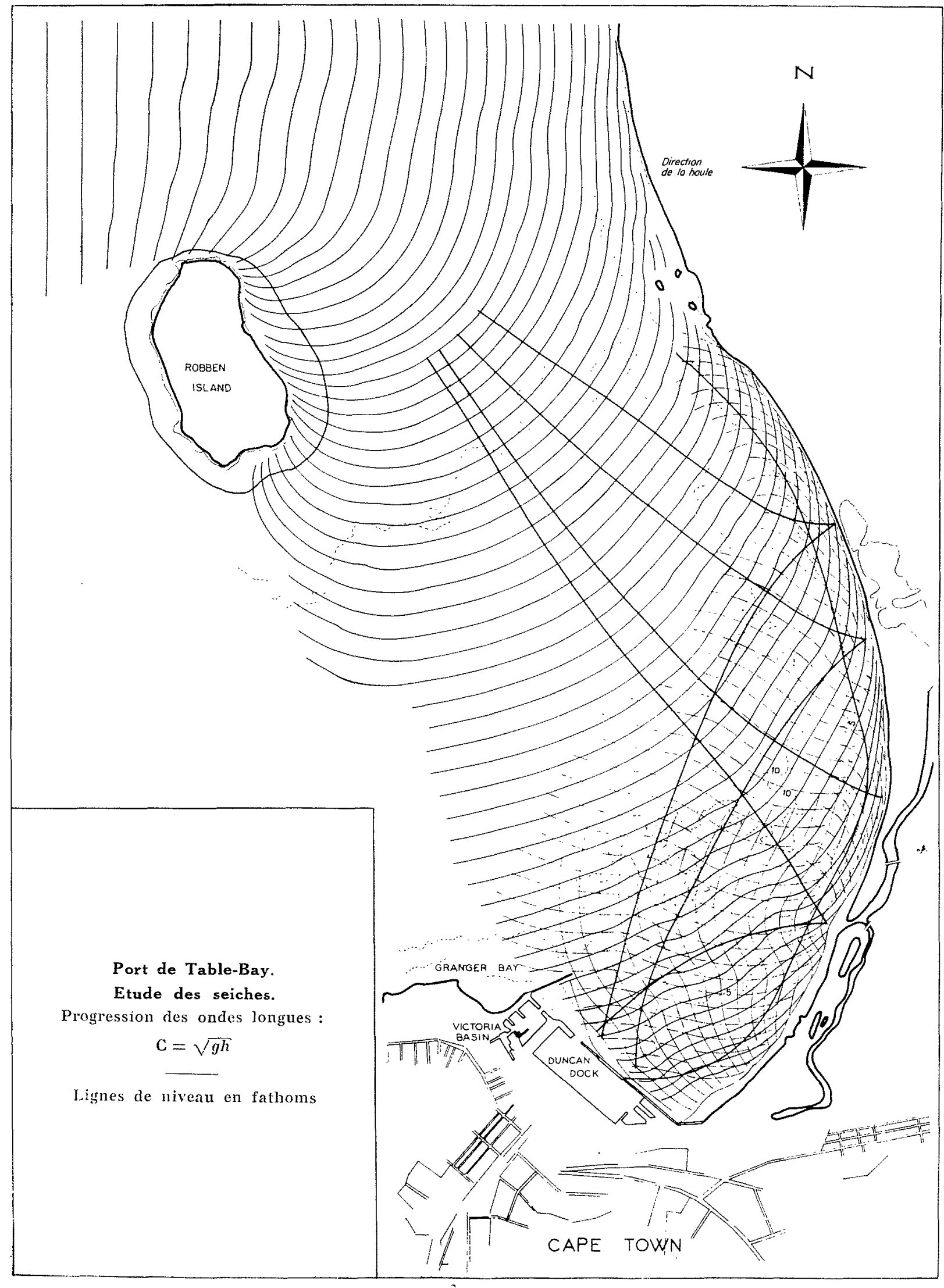

Fig. 2. - Le port du Cap est un exemple possible de « miroir concave $»$. Les ondes de seiches après diffraction autour de Robben Island viennent se réfléchir sur la côte de la baie de la Table. L'énergie réfléchie se concentre à l'entrée des bassins et l'agitation est encore amplifiée par la présence de la jetée située au nord du bassin Victoria. 
nent. En pratique, la complexité des phénomènes est telle qu'il est sage de se limiter à des déductions relativement simples comme celles que nous avons faites à propos de la côte des
Landes. Si nous avons abordé le problème d'ensemble, ce n'est que pour mettre la diseussion des études sur modèle dans le cadre et, dirionsnous presque, l'ambiance nécessaire.

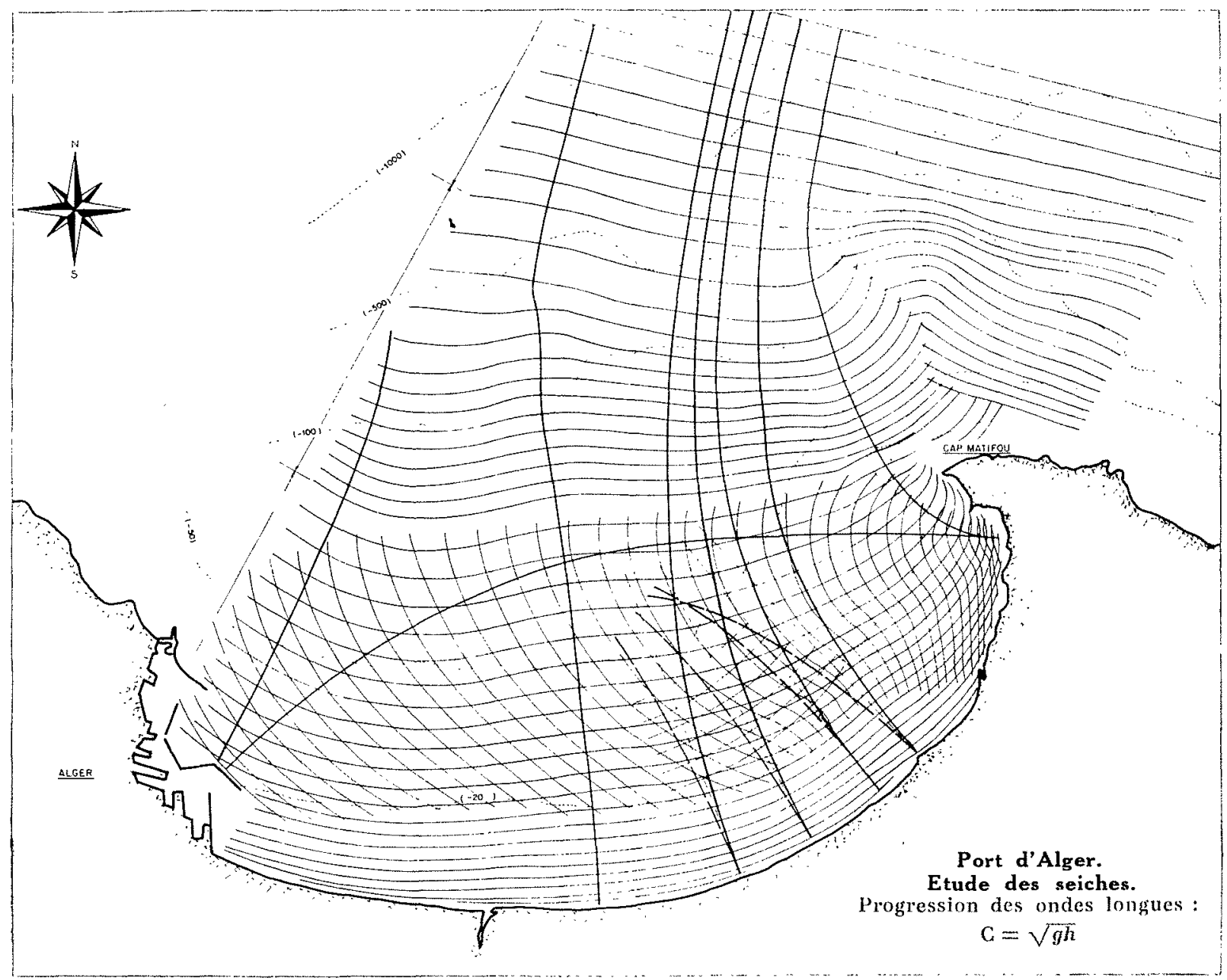

Fia. 3. - La baie d'Alger se présente aussi comme un miroir concave pour les ondes de seiches. La comparaison entre les figures 2 et 3 permet d'appréeier la grande influence de la présence d'une île comme Robben Island sur la propagation des ondes génératrices de seiches.

\section{IV. - DIFFERENCES ESSENTIELLES ENTRE LES ETUDES SUR MODELE REDUIT DES SEICHES ET DES HOULES}

Ces différences sont nombreuses et profondes, sans entrer dans trop de détails nous en donnons ci-dessous les principales.

\section{1. -- Etudes pRÉliminaires.}

Lorsque l'on fait le projet d'un modèle réduit destiné à l'étude de la pénétration de la houle dans un port, une des premières étapes est de faire un « plan de houle » (diagramme de réfraction) de façon à savoir quelles positions et quelles orientations il est nécessaire de donner au batteur. Parfois, on peut placer ce dernier par des profondeurs lelles que la houle commence à peine à tourner el par conséquent présente encore un front rectiligne.

$\mathrm{Ce}$ cas ne se produira jamais pour des seiches $\left(^{\star}\right)$. Car, ainsi que nous l'avons vu, celles-ci auront toujours subi des effets de

$\left(^{*}\right)$ Nous exceptons bien entendu le cas où la seiche est directement imputable aux houles. 
réfraction notables. Il faudra néanmoins placer le batteur en un endroit où les crêtes, quoique réfractées, seront encore à peu près rectilignes (à moins d'utiliser un batteur serpent [2]). Pour chaque cas particulier il est nécessaire de faire des épures de réfraction spéciales, mais il est cependant possible de faire quelques remarques générales.

Les ondes de seiches étant réfractées même par les plus grands fonds océaniques, l'épure de réfraction doit donc commencer en plein océan; en fait elle doit couvrir tout l'espace entre le point d'origine supposé et le point d'arrivée (fig. 4). Des épures à une telle échelle ne sont pas sans poser des problèmes inédits car, en toute rigueur, il est nécessaire de tenir compte de la courbure de la terre et de l'accélération de Coriolis. Toutefois des études théoriques semblent montrer que cette dernière a une influence pratiquement négligeable $\left(^{*}\right)$.

${ }^{*}$ ) On sait que d'après certaines hypothèses les ondes de seiches peuvent être dues au déferlement sur la côte de houles irrégulières émettant des ondes longues vers le large ayant la période des trains de houle. On est alors conduit à tracer l'épure des ondes incidentes pour une période dont l'ordre de grandeur est celui de la houle. Par contre l'épure des ondes réfléchies doit être tracée pour la période des trains de houle.

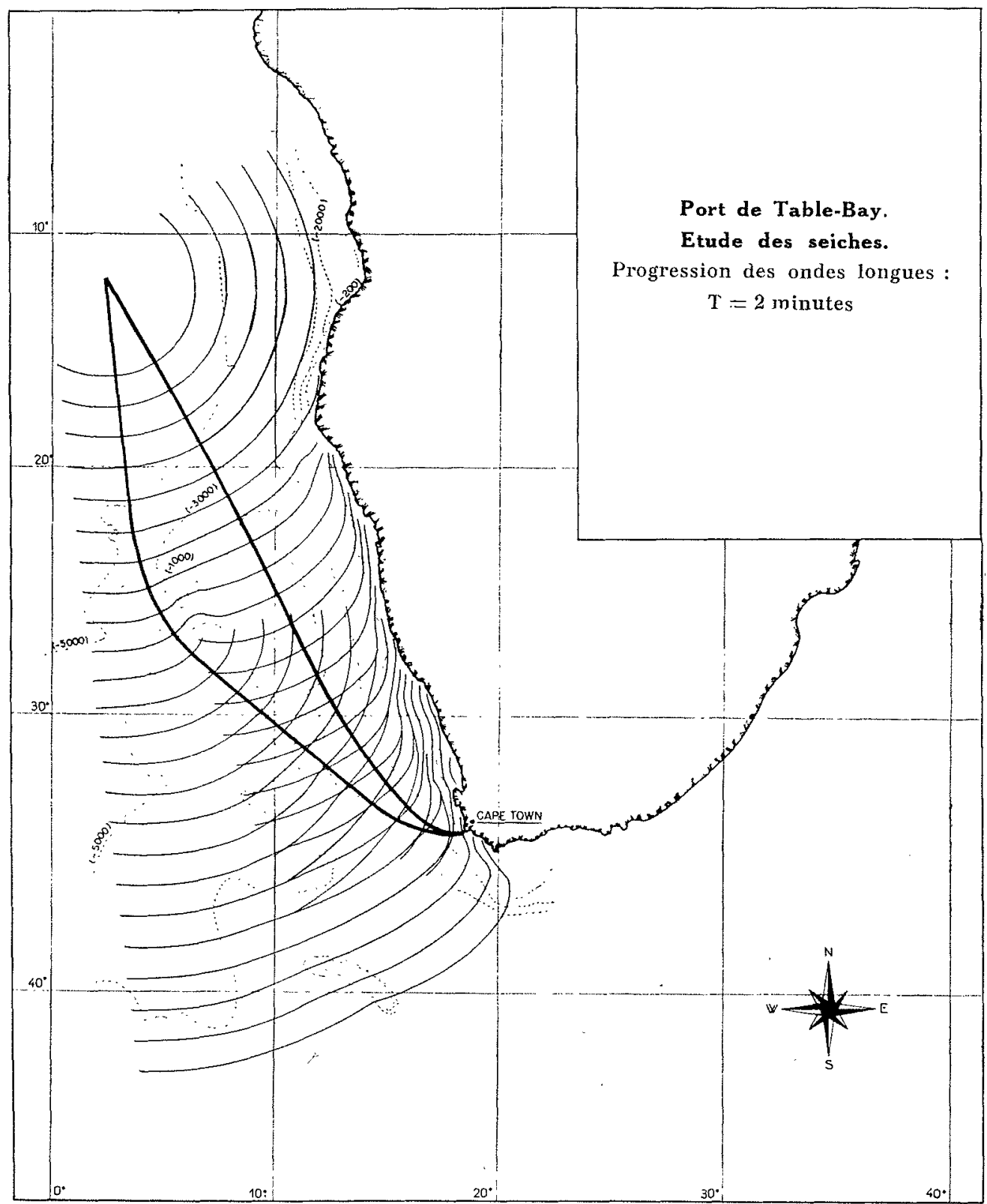

FIG. 4. - Les ondes de seiches étant réfractées même par les plus grands fonds océaniques, l'épure de réfraction doit donc commencer en plein océan. En fait elle doit couvrir tout l'espace entre le point d'origine supposé et le point d'arrivéc. 
Les lois de la réfraction sont telles que les conclusions que l'on déduira de telles épures présenteront en général un certain nombre de caractères communs :

Lorsque le fond n'est pas trop tourmenté, en particulier lorsque les lignes de sonde sont grosso modo parallèles à la côte, les crêtes sont toujours pratiquement parallèles au rivage lorsqu'elles atteignent des profondeurs propres à l'établissement d'un port (fig. 5). Dans le cas idéal où les lignes de sonde sont parallèles à la côte on peut calculer qu'une onde de $2 \mathrm{mn}$ émise par grands fonds (4.000 $\mathrm{m}$ par exemple) ne peut avoir un angle d'incidence supérieur à environ :

$$
\begin{array}{rrr}
20^{\circ} & \text { par } & 200 \mathrm{~m} \text { de fond } \\
11^{\circ} & \text { par } & 100 \mathrm{~m} \text { de fond } \\
5^{\circ}, & \text { par } & 20 \mathrm{~m} \text { de fond } \\
3^{\circ} 30^{\prime} \text { par } & 10 \mathrm{~m} \text { de fond }
\end{array}
$$

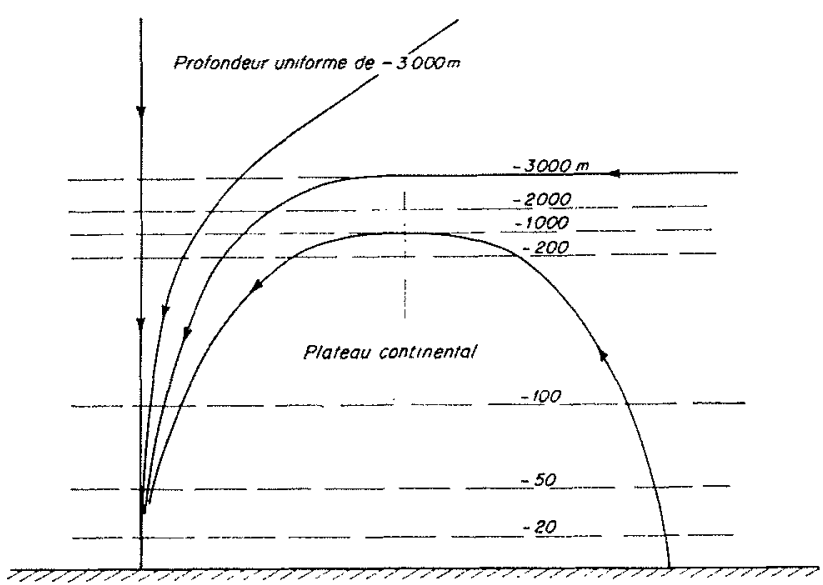

Fig. 5. - Les crêtes d'onde de seiche sont toujours pratiquement parallèles au rivage lorsqu'elles atteignent des profondeurs propres à l'établissement d'un port.

Si l'on se limite à l'hypothèse que les ondes de seiches viennent du large on voit qu'il sera en général suffisant d'avoir un générateur de direction fixe. Dins celte hypothèse, que nous avons adoptée ici, rappelons-le, pour limiter la discussion, il n'est pas licite d'étudier des excitations sous des incidences quelconques.

\section{2. - Influence du plateau continental.}

Les terres émergées ne sont pas les seules a réfléchir les ondes de seiches. Les épures de réfraction de ces ondes risquent donc d'être particulièrement délicates. Sans vouloir traiter complètement cette question, il est cependant particulièrement utile de la considérer en ce qui concerne l'action possible du plateau continental.
Considérons tout d'abord l'épure inverse (*) de celle déjà considérée, e'est-à-dire la propagation d'une onde de seiche à partir d'un point de la côte (fig. 6).

Supposons par exemple qu'une baie soit en résonance et que son entrée émette des ondes à peu près circulaires par $20 \mathrm{~m}$ de fond par exemple. On voit que seule l'énergie émise dans un angle de $10^{\circ}$ (soit environ $5 \%$ de l'énergie si l'émission n'est pas directionnelle) a des chances de s'échapper dans l'océan. Le reste est ramené vers la côte par un phénomène de « réflexion totale $\gg$ se produisant principalement sur la limite extérieure du plateau continental. On voit done que cette limite et la côte peuvent se renvoyer les ondes de seiches de nombreuses fois et ainsi créer des phénomènes de résonance non négligeables. Le mécanisme de la réfraction n'est. d'ailleurs pas le seul susceptible de produire des phénomènes analogues $\left.{ }^{* \star}\right)$.

Lorsque la limite du plateau continental sera très éloignée de la côte il ne sera pas possible de la représenter sur un modèle d'échelle raisonnable, mais, d'autre part les phénomènes de résonance seront moins nets et il suffira en général d'un examen sur plan pour étudier leur influence possible. Par contre lorsque le plateau continental sera relativement étroit il pourra jouer un rôle important dans la sélection des fréquences dangereuses et il sera indispensable d'étudier son influence avec soin. Pour des formes de côtes et de plateau continentaux compliquées il pourra même être nécessaire de faire un modèle préliminaire spécial représentant à très petite échelle une vaste portion de côte englobant le plateau continental et une étendue suffisante des profondeurs abyssales avoisinantes.

Ce dernier type de modèle pose lui aussi certains problemes de similitude, choix des ćchelles, etc..., que nous n'abordons pas ici.

\section{3. -- ETENDUE DE LA RÉgION A HEPRÉSEN'TER SUR LE MODELE PROPREMLNT DIT. -- ECHYLLT: EN PLAN.}

Nous avons vu combien les ondes de seiche sc réfléchissaient facilement.

De vastes baies, bordées de plages ou non, peuvent donc former caisse de résonance pour des ondes de seiches incidentes et ainsi que

(*) Une épure « inverse » est souvent le meilleur moyen de se rendre compte des iucidences maxima possibles, suivant lesquelles l'onde de seiche venant du large peut atteindre la côte. Réciproquement elle visualise la quantité d'énergie qui peut être émise d'un point de la côte.

$\left(^{*}\right)$ Nous savons par exemple que le plateau continental peut rentrer en résonance lorsque le mouvement est bidimensionnel [6]. 


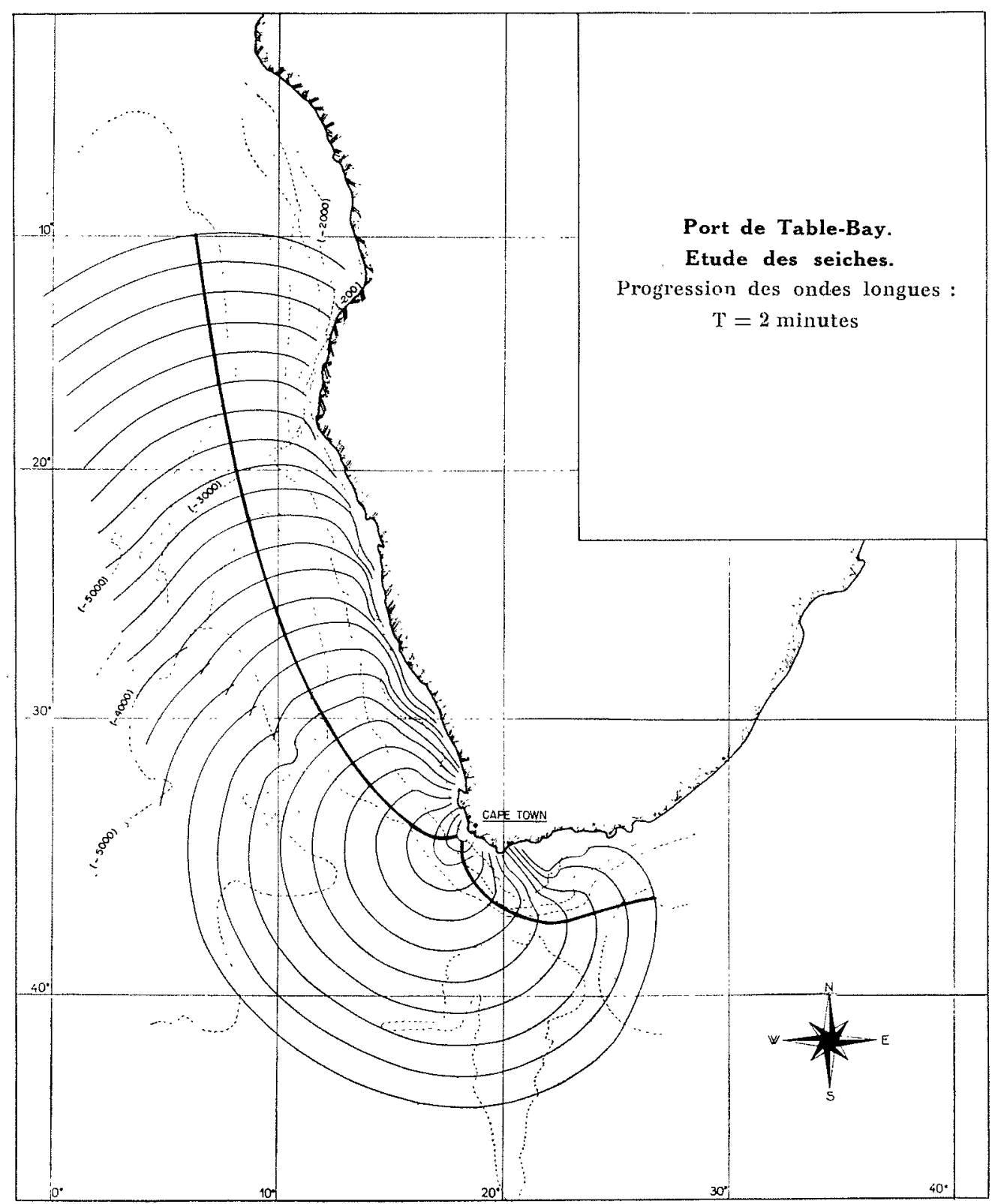

Fir. 6. - Une épure « inverse » est souvent le meilleur moyen de se rendre compte des incidences maximum possibles, suivant lesquelles l'onde de seiche venant du large peut atteindre la côte.

nous l'avons vu plus haut (exemple de la côte des Landes, du port d'Alger ou de Table Bay) des côtes étendues peuvent former des réflecteurs faisant converger les ondes sur certains points particuliers.

Là encore, les épures, et plus généralement les études sur plan permettent le plus souvent de limiter les régions à représenter sur modèle. Toutefois ces régions seront en général plus importantes que celles qu'il est nécessaire de considérer pour les modèles à houle. Lorsqu'un port sera par exemple situé en un point d'une baie, il pourra être nécessaire de représenter la totalité de celle-ci même si son étendue se chiffre par kilomètres, voire même par dizaines de kilomètres.

D'autre part, la nécessité de représenter les fonds jusqu'à une zone où les crêtes sont suffisamment rectilignes conduira à agrandir le modèle vers le large au-delà des accidents locaux qui pourront provoquer des réfractions ou des diffractions importantes (hauts fonds, promontoires, îles, etc...).

Il est important d'insister ici sur le fait que l'étude d'un port ne peut pas en général être détachée de celle du «milieu » océanique où il se trouve. Il serait évidemment extrêmement économique de pouvoir se limiter à l'étude du 
" problème intérieur », le modèle se bornant alors à représenter le port et l'excitation étant fournie à l'entrée ou à son voisinage immédiat par un dispositif convenable.

Ce genre de disposition permet effectivement de déceler les modes de résonance propre du port, mais il ne permet pas en général de conclure sur leurs amplitudes. En effet celles-ci dépendent directement de l'amplitude de l'agitation au droit de l'entrée du port et cette agitation a une valeur locale qui dépend essentiellement du mode d'agitation extérieur, fonction luimême de la topographie des grands espaces entourant le port.

Dans un grand port on ne pourra supprimer tous les modes d'oscillations indiqués par ce genre d'essais qu'en barrant le plan d'eau dans tous les sens, ce qui créerait (à grands frais) des obstacles inadmissibles pour la navigation. D'autre part, si l'on se limite à des ouvrages ne visant qu'à supprimer quelques modes d'oscillation, on risque de laisser passer et même de renforcer d'autres modes déjà amplifiés par les interférences ou résonances extérieures au port. A ce propos, il est intéressant de signaler qu'il est parfois possible d'étudier séparément la résonance des plans d'eau extérieurs et celle du port. Ce cas se présente lorsque l'entrée du port est suffisamment petite pour que, d'une part, l'état de résonance intérieure n'influe pas trop sur la configuration de l'agitation extérieure et que, d'autre part, l'agitation intérieure ne dépende pratiquement que de la valeur locale de l'amplitude verticale extérieure au centre de l'entrée.

Le problème extérieur est alors étudié sans que l'on ait le souci d'assurer une similitude convenable pour les bassins du port dont la profondeur est relativement faible. Ceci permet de travailler à une échelle plus petite. On détermine sur ce modèle le « coefficient d'amplification » du plan d'eau extérieur, c'est-à-dire le quotient de l'amplitude incidente.

On étudie ensuite le problème intérieur et on définit de nouveaux « coefficients d'amplification 》qui sont les quotients des amplitudes relevées aux différents points de mesure par l'amplitude à l'entrée. Cette deuxième étude peut se faire à une échelle relativement importante sans exiger un modèle très grand et coûteux. Elle permet des mesures précises ainsi que l'étude commode et détaillée des modifications à apporter aux installations intérieures du port.

On obtient ensuite pour chaque fréquence et pour chaque point de mesure le coefficient d'amplification global en faisant le produit des coefficients intérieurs et extérieurs. (Ceci suppose négligeable les effets de turbulence.)

En résumé, nous voyons que l'étude sur mo- dèle ne peut se limiter au problème intérieur que dans des cas exceptionnels. Cela sera admissible soit pour des ports ne recevant que des bateaux de faible tonnage où il sera possible, sans inconvénients majeurs, de diviser le plan d'eau en darses de dimensions limitées, soit lorsque la topographie extérieure sera si simple que l'on pourra déterminer sur plan les caractéristiques de l'agitation extérieure soit encore lorsqu'un modèle préliminaire aura déterminé les « coefficients d'amplification » extérieurs.

Dans le cas général, l'étendue des régions à représenter sur le modèle sera donc beaucoup plus importante que dans les études de houle. Heureusement la grande longueur des ondes de seiche autorise l'emploi d'échelles en plan très petites, de l'ordre du dixième de celles employées pour les modèles à houle. De plus il est possible d'utiliser l'artifice de la distorsion et ainsi d'éviter de travailler avec des nappes d'eau trop minces.

\section{4. - DistorsION DES FONDS.}

L'étendue des régions à représenter rend souhaitable de pouvoir utiliser une échelle en plan de l'ordre $\mathrm{du} 1 / 1.000$ ou du 1/2.000, par exemple.

Si on employait la même échelle verticale, les profondeurs de $10 \mathrm{~m}$, courantes dans les grands ports seraient représentées sur le modèle par des couches de 0,5 à $1 \mathrm{~cm}$ où les frottements seraient manifestement trop importants. On sait que ceux-ci peuvent être réduits grâce à la dislorsion; leur importance dans la nature étant négligeable, plus la distorsion sera grande, plus la similitude des frottements sera respectée. Il est donc essentiel d'examiner si l'on peut distordre et quel degré de distorsion est compatible avec la similitude.

On sait qu'il n'est pas admissible, en général, de distordre un modèle destiné à l'étude de la pénétration de la houle dans un port. La principale objection est que la similitude de réfraction n'est pas conservée. D'autre part la similitude des pouvoirs réfléchissants est également altérée.

En ce qui concerne ce dernier point, nous aurons l'occasion d'y revenir. Pour le premier point, il importe de mentionner que la similitude de réfraction peut néanmoins être conservée lorsque la longueur des ondes est suffisamment grande par rapport à la profondeur maximum de la région étudiée pour que la célérité soit donnée avec une approximation satisfaisante par la formule $c=\sqrt{g h}$. On peut élargir un peu ce domaine d'utilisation de la distorsion par l'application de la distorsion 
fonctionnelle imaginée par M. CarLotTr (*) mais supposons tout d'abord que l'on emploie la distorsion ordinaire. La formule $c=\sqrt{g h}$ est valable à $5 \%$ près environ si l'on a :

$$
h_{m n}<6 \mathrm{~T}_{s}^{2}
$$

En mer, pour des ondes de $2 \mathrm{mn}$ de période, la formule $\sqrt{g h}$ sera donc valable (à $5 \%$ près) jusqu'à des fonds de :

$$
6 \times 14.400=86.400 \mathrm{~cm} \text { soit } 864 \mathrm{~m}
$$

Sur un modèle distordu (1) d'échelles horizonlale $\lambda$ et verticale $u$. (échelle des temps $\lambda / \sqrt{\mu \text { ) la }}$ même relation rapportée aux grandeurs réelles $(h$ et $\mathrm{T}$ ) donne :

$$
h<6\left(\frac{\lambda}{\mu}\right) \mathrm{T}^{2}, \text { soit } h<6(\delta)^{2} \mathrm{~T}^{2}
$$

ò étant la distorsion. La profondeur réelle maximum qui peut être représentée par le modèle distordu est donc inversement proportionnelle au carré de la distorsion. Pour une distorsion de 2 elle serait, dans le cas précédent, de $216 \mathrm{~m}$ seulement ce qui sera très souvent suffisant. Pour une distorsion de 10 elle ne serait plus que de $8,64 \mathrm{~m}$, ce qui serait certainement insuffisant.

La formule ci-dessus permet une instructive discussion générale. Nous supposerons pour simplifier que l'étendue des fonds à représenter soit déterminée par des considérations de topographie, ou autres, et que la plus grande valeur de la profondeur soit ainsi une des données du problème.

Nous supposerons encore que l'on s'impose une échelle verticale (minimum) y. de façon à ce que les plans d'eau de l'ordre de $10 \mathrm{~m}$ soient représentés par une épaisseur suffisante sur le modèle. Il reste alors :

$$
\mathrm{T}^{2} \lambda^{2}>\frac{h \mu^{2}}{6}
$$

On voit que plus la période des seicnes sera grande, plus l'échelle horizontale pourra être petite. D'autre part, plus la période est grande plus la longueur d'onde est grande et nous avons vu que l'étendue des régions à représenter était en général proportionnée à cette dernière. (Nous verrons plus loin que c'est aussi le cas des zones du modèle nécessaires à l'amortissement des ondes qui, dans la nature, repartent vers le large.) Il y a ainsi une sorte de compensation, l'échelle pouvant diminuer lorsque l'étendue à représenter doit augmenter.

${ }^{*}$ ) Nous supposons dans ce qui suit que l'on considère des distorsions assez marquées, par exemple, supérieures à 1,5 pour fixer les idées [3].
On voit que la conception d'un modèle dépend étroitement de la période des seiches que l'on veut étudier. Si l'on veut étudier une gamme de périodes étendues, il pourra donc être économique de faire plusieurs modèles de façon à éviter l'obligation de reproduire les grandes étendues nécessaires pour les périodes les plus longues à la grande échelle nécessaire pour les périodes les plus courtes.

Quelques exemples numériques permettront de préciser ce qui précède. Supposons que l'on veuille étudier des seiches relativement courtes de période $1 \mathrm{mn}$ et qu'il soit nécessaire de représenter une région allant jusqu'à des profondeurs de l'ordre de $100 \mathrm{~m}$ par exemple.

La distorsion maximum utilisable est alors de l'ordre de 1,5. Si l'échelle verticale choisie est de 1/400, l'échelle en plan sera de 1/600.

Pour des seiches de 2 mn il pourra être nécessaire d'aller jusqu'à une profondeur plus grande, par exemple $150 \mathrm{~m}$. La distorsion pourra alors être de 3 , ce qui donne avec la même échelle verticale, une échelle en plan de 1/1.200.

Si l'on veut étudier des seiches de $4 \mathrm{mn}$, la profondeur maximum étant de $200 \mathrm{~m}$ par exemple, on pourra utiliser une distorsion de 4. Avec la même échelle verticale on peut donc utiliser une échelle de 1/1.600. Cette échelle plus réduite pourra éventuellement permettre de loger ce modèle dans le même espace que le précédent quoique la région représentée soit plus vaste et que les organes accessoires du modèle soient relativement plus importants.

Si l'on a, a priori, des raisons de penser que les périodes les plus dangereuses sont comprises dans une gamme relativement étroite (par exemple par suite de la valeur de la période de résonance des navires amarrés), il est possible de se limiter à un modèle qui sera optimum pour une période moyenne bien choisie. $\mathrm{Ce}$ modèle pourra néanmoins servir à étudier approximativement les résonances des périodes assez différentes, quoique dans ce cas, il ne donnera que des indications sur l'existence possible d'autres gammes de fréquences dangereuses qui, si elles semblent inquiétantes, pourront justifier la modification du modèle.

I'emploi de la distorsion fonctionnelle permet, toutes choses ëgales d'ailleurs, de réduire l'échelle horizontale des modèles en autorisant des distorsions plus importantes. Elle a également l'inconvénient d'être essentiellement adaptée à une gamme de période, assez étroite, et de plus à des niveaux de marée pas trop variables.

\section{5. - Similitude des Réfiexions. - Distorsion D'AMPLITUDE DES ONDES DE SEICHE.}

Quoique très différentes ces deux questions sont étroitement liées. On sait, en effet, que Je 
coefficient de réflexion d'un ouvrage, d'une plage, etc..., est essentiellement fonction :

de la pente de l'ouvrage,

de la cambrure de la houle, de l'échelle.

La distorsion des profondeurs accroît les pentes et par conséquent les réflexions. L'augmentation de cambrure des houles réduit les réflexions. La réduction d'échelle accroît les réflexions.

Ce dernier eff et est relativement moins important; c'est le seul qui joue dans les modèles d'étude de la houle où la distorsion des profondeurs est le plus souvent proserite et où la distorsion (en plus ou en moins) des amplitudes peut, en général, être évitée (sur les inconvénients de cette distorsion du point de vue de la similitude des réflexions voir l'article [4] de MM. Greslou et Mahé déjà cité).

Au contraire, les modèles d'étude des seiches seront, en général, distordus tant du point de vue des profondeurs que du point de vue des cambrures étant donné l'extrême petitesse de ces dernières dans la nature. Les effets de ces deux distorsions étant opposées, on peut espérer corriger l'un par l'autre. Examinons cette idée.

Nous avons vu plus haut que la cambrure limite au large des ondes pouvant se réfléchir intégralement sur une pente était donnée par :

x. étant petit.

$$
\gamma=0,254.5 / 2
$$

Pour $\gamma=10^{-6}$, on avait $\alpha=0,0083$. Si l'on considère l'exemple moyen donné plus haul (échelle verticale $1 / 400$ - échelle horizontale $1 / 1.200$ ) on voit que la distorsion des cambrures (en profondeur infinie) devrait être : $(3)^{5 / 2}=15,6$. Mais sur un modèle distordu, le «gonflement» de la houle est moindre pour des profondeurs homologues (les cambrures en profondeur relative faible sont diminuées dans le rapport (o) $-3 / 2$ si la cambrure en eau profonde est la mène).

En conséquence, la distorsion des cambrures dans la zone utile ne devrait être que de 3 . L'échelle verticale des amplitudes serait donc de $3 / 1.200$ soit $1 / 400$. Une amplitude de $20 \mathrm{~cm}$ (par faibles profondeurs) serait représentée sur le modèle par $0,5 \mathrm{~mm}$.

Le calcul qui précède tend donc à montrer que l'on peut compenser les effets de la distorsion des profondeurs par une distorsion des amplitudes et même que la distorsion à laquelle on aboutit ainsi est relativement peu importante et sera souvent insuffisante pour assurer une précision de mesure convenable.

En fait, il est justifié d'augmenter quelque peu la distorsion à laquelle nous venons d'abou- lir pour tenir comple également de l'effet d'échelle, mais même ainsi, on risque d'être conduit à utiliser des amplitudes très faibles.

C'est pourquoi il peut ètre intéressant d'augmenter encore la distorsion et ainsi de travailler avec des amplitudes très raisonnables, grâce à des artifices permettant de restituer des coefficients de réflexion corrects. Dans ce but, une plage pourra, par exemple, être représentée de la façon indiquée par la figure 7 . Cet artifice introduit lui-même une cause d'erreur car il n'est pas possible de choisir les proportions de façon à ce que les phases des réffexions soient reproduites fidèlement à la fois pour les ondes normales et pour les ondes obliques $\left(^{\star}\right)$. Il ne faudra donc pas en user sans nécessité, autrement dit, il ne faudra pas exagérer inutilement la distorsion des amplitudes ${ }^{\star *}$ ).

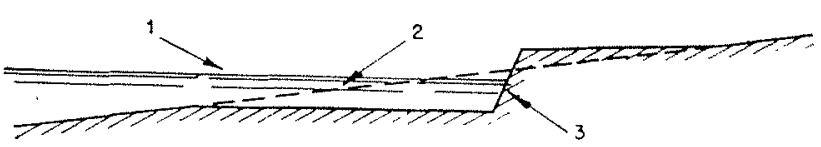

FIG. 7. - Dispositif permettant de respecter la similitude de la réflexion des ondes de seiches.

Il faut souligner également que le taux d'amortissement des ondes liquides croît avec leur cambrure (à partir d'une certaine valeur de celle-ci) par suite de phénomènes de turbulence. Nous avons vu [1] à quel point le coefficient d'amplification de résonance peut être sensible à une augmentation de cambrure de la houle incidente. Ceci conduit donc à fixer unc limite à l'exagération des amplitudes, mais cette limite est en général supérieure à celle résultant des considérations des paragraphes précédents.

\section{6. - CONDITIONS ACX Limites, ABSORBJURS, FILTRES.}

On sait que les parois des bassins d'essais qui limitent les modèles à houle ver's le large, ne doivent pas être la cause de réflexions parasites n'ayant aucun rapport avec la réalité. On est donc conduit à disposer des absorbeurs de vagues sur toutes les parois qui n'ont pas d'homologue dans la nature. Dans le même ordre d'idée, il est nécessaire de placer un «filtre » absorbant devant le batteur pour minimiser les réflexions parasites qui peuvent se produire sur

(*) De plus, comme nous l'a fait xemarquer M. J. Forr du L.N.H., ce dispositif ne peut convenir lorsque le port est implanté sur une côte sonmise à unc maréc de forte amplitude.

$\left(^{*}\right)$ On peut signaler que les cambrures étant plus faibles que sur les modèles a houle, il sera en général possible d'étudier l'agitation par la méthode du ciel étoile de M. Barilion. 
celui-ci. On évite également ainsi les phénomènes de résonance propres à l'ensemble du modèle.

En première approximation, on peut dire que la largeur minimum des plages absorbantes ou des filtres est proportionnelle à la longueur d'onde (valeur locale dans le modèle), un ordre de grandeur raisonnable étant une longueur d'onde. Il en résulte que les filtres et amortisseurs qui sont un accessoire relativement peu encombrant sur les modèles à houle deviennent énormes sur les modèles de sciche du fait de l'importance des longueurs d'ondes. Il arrivera souvent que ces organes occupent de beaucoup la plus grande partie du bassin d'essais et la caractéristique la plus frappante d'un modèle de seiches bien conçu est l'exiguité presque ridicule du port par rapport à l'étendue de l'ensemble.

Nous avons vu plus haut que la distorsion de cambrure des ondes de seiches ne devait pas être trop exagérée. Par conséquent, même sur le modèle, on aura des ondes non seulement longues mais également peu cambrées. Des amortisseurs constitués par des plages devraient avoir des pentes très douces et par conséquent ètre extrêmement longs et encombrants pour être efficaces. Le déferlement pur et simple étant défaillant, on fera appel à l'amortissement par perméabilité, rugosité, résonance, déversement, etc... Sauf peut-être avec ce dernier procédé qui est actuellement en cours d'étude au Laboratoire, il semble bien nécessaire de disposer d'au moins une longueur d'onde pour réaliser un amortissement suffisamment efficace.

Un problème analogue se pose pour les filtres qui doivent être beaucoup plus efficaces que sur un modèle à houle du fait des forts coefficients de réflexion des ondes de seiches. D'ailleurs la faible cambrure requise permet des filtrations énergiques. Là encore, il faut que les filtres règnent sur à peu près une longueur d'onde car une action trop brutale créerait des réflexions plus grandes que celles que l'on veut éviter. Des perfectionnements, tels que le filtre progressif étudié par M. LE MéHauté [10] peuvent permettre d'économiser de la place sans altérer la qualité du résultat.

La figure 8 est un plan schématique d'un modèle correspondant aux caractéristiques étudiées plus haut.

Un modèle de seiche sera donc essentiellement caractérisé par l'importance des dispositifs propres à respecter les conditions aux limites. L'épaisseur des filtres à houle et des ouvrages absorbants est de l'ordre de grandeur d'une longueur d'onde.

Le générateur d'onde peut occuper une position fixe déterminée par une épure inverse. (Nous exceptons, bien entendu, les hypothèses

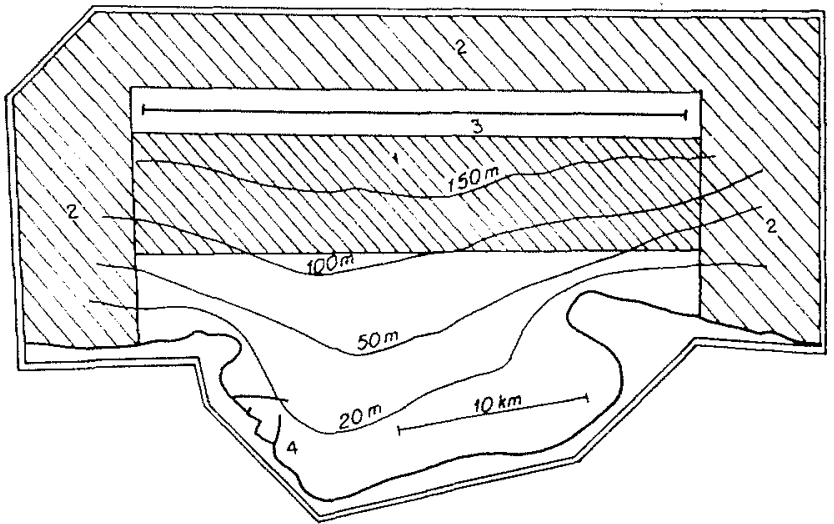

Fig. 8. - Un modèle de seiche sera essentiellement caractérisé par l'importance des dispositifs propres à respecter les conditions aux limites: filtres à houle et ouvrages absorbants. Ce modèle est prévu pour étudier les seiches de période voisine de $2 \mathrm{~min}$.

d'après lesquelles l'onde de seiche est due dans la nature aux houles irrégulières. Il peut être alors nécessaire de prévoir sur le modèle plusieurs positions pour le générateur d'onde.)

On voit l'encombrement énorme de l'ensemble anortisseurs-filtres batteurs qui est indispensable à la réalisation correcte des conditions aux limites.

\section{7. - RÉglage des Caractéristioues de L'ONdE.}

Direction. - Par suite de la largeur des filtres, l'emplacement du batteur correspond parfois à des profondeurs considérables qu'il ne serait pas commode de représenter à l'échelle verticale choísie sur un modèle usuel.

Ainsi que pour les modèles de houle on arrête donc, en général, la représentation des fonds au-delà d'une certaine cote à laquelle se trouve une dalle horizontale supportant le batteur. Sur les modèles de seiches il ne faut pas oublier que cette modification des fonds fausse la réfraction et que par conséquent il ne faut pas donner au batteur l'orientation des crêtes qui correspond à sa position géographique, mais une orientation déduite par une épure inverse à partir du plan de vague à réaliser au-dessus des fonds représentés.

Amplitude. - On sait combien la présence d'ondes réftéchies par le batteur (après avoir été déjà réfléchies par le modèle) est gênante pour la mesure précise de l'amplitude incidente dans les modèles de houlè à trois dimensions. Les filtrcs employés dans ce type d'étude ne sont pas très puissants (car ils doivent laisser passer une houle de cambrure notable), ils sont donc incapables de rendre complètement négligeable l'influence des réflexions multiples. 
Le même genre de difficulté se retrouve pour les modèles de seiches avec la différence, cependant, que la puissance des filtres n'est limitée en pratique que par la place disponible pour les mettre. Quoique l'énergie réfléchie par le modèle représente une fraction relativement importante de l'énergie incidente, il est donc théoriquement possible de réduire beaucoup les perturbations sur le batteur. En résumé, si l'on utilise un filtrage énergique, on peut étalonner le batteur en émission sur un amortisseur parfait et se servir de cet étalonnage au cours des essais, chose souvent impossible sans précautions spéciales dans le cas des modèles à houle.

FrÉQuence. - Si pour l'amplitude nous rencontrons une facilité relative, il n'en est plus de même en ce qui concerne le réglage de la fréquence.

On sait, en effet, que les phénomènes de diffractions, réfractions, déferlement, etc..., varient d'une façon continue et régulière avec la fréquence mais qu'il n'en est pas de même pour les phénomènes de résonance. Rappelons par exemple à ce sujet l'étude $d u$ professeur Mc Nown, effectuée au Laboratoire Dauphinois d'Hydraulique, sur les oscillations des eaux portuaires dans un port circulaire où il a montré que dans certains cas des variations relatives de fréquences de l'ordre du $1 / 1.000$ pouvaient changer du tout au tout l'organisation de l'agitation, substituant des ventres aux nouds, etc... (fig. 9) [7 et 8 ].

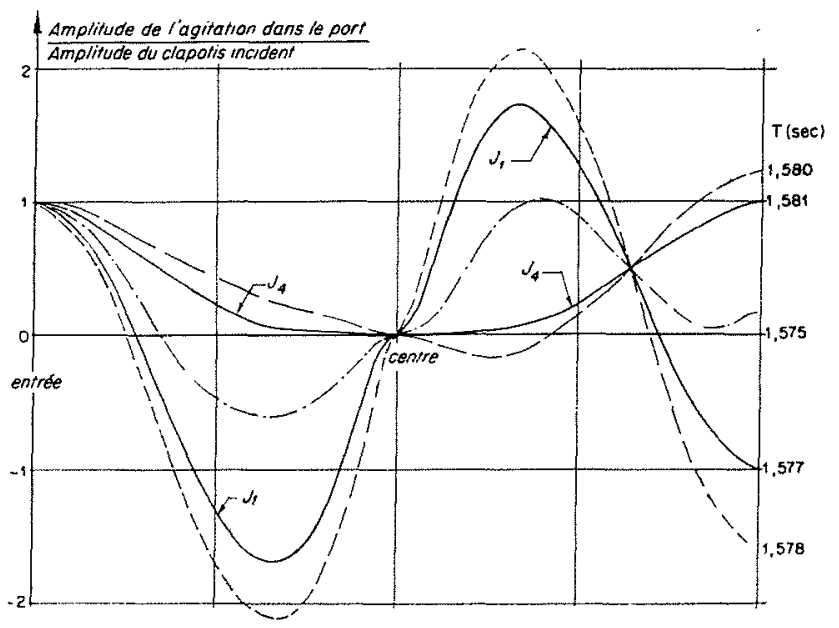

FIG. 9. - Des variations relatives de fréquences de l'excitation de l'ordre du $1 / 1.000$ peuvent changer du tout au tout l'organisation de l'agitation à l'intérieur des ports, substituant des ventres aux nœuds... Ces courbes représentent une forme de l'agitation dans un port circulaire, suivant un diamètre, en fonction de la période du mouvement.
Cette sensibilité de l'agitation à des variations infimes de fréquences se manifeste d'autant plus que les limites du plan d'eau sont plus réfléchissantes et que la longueur d'onde est plus petite par rapport aux dimensions des régions susceptibles d'entrer en résonance.

Or nous savons que pour les seiches presque toutes les limites naturelles du plan d'eau sont parfaitement réfléchissantes et, d'autre part, que les régions à représenter sont parfois très grandes.

Un port débouchant dans une baie assez fermée sera donc soumis à une agitation de seiches pouvant varier brutalement pour de faibles variations de fréquences. L'intérieur du port lui-même pourra répondre de façons très différentes à des fréquences très voisines pourvu que ses dimensions soient assez grandes par rapport à la valeur locale de la longueur d'onde (des dimensions linéaires de quelques longueurs d'onde suffisent).

Il est à noter que si le port débouche dans une baie largement ouverte (ou d fortiori sur une côte rectiligne), la limite côté large de la baie sera non réfléchissante et par conséquent les effets d'instabilité que nous venons d'étudier seront atténués.

Au contraire si on négligeait de garnir d'amortisseurs les murettes d'un bassin d'essai de seiches, et de filtres son générateur, on aurait constamment des systèmes quasi stationnaires se permutant l'un l'autre sous le moindre prétexte. Non seulement les essais seraient inutilement compliqués mais surtout ils perdraient toute signification physique.

Les résultats des essais peuvent donc être très différents pour des fréquences très voisines. On est ainsi amené aux conclusions suivantes :

a) Les essais à fréquence fixe doivent se faire avec une fréquence d'une constance extrême. Il n'est pas possible d'indiquer d'une façon générale la précision qui doit être atteinte car celle-ci dépend dans chaque cas des formes géométriques du plan d'eau et de la fréquence elle-même. Il arrivera même que les mouvements d'une fréquence donnée ne puissent être stabilisés quelles que soient les précautions prises en vue du réglage de la fréquence. (Existence de deux périodes de résonance extrêmement voisines mais correspondant à des agitations très différentes.) Si la fréquence est réglée soigneusement (par exemple au $1 / 1.000$ ), ces accidents n'arriveront pas trop souvent et n'entacheront guère la validité d'ensemble des résultats. Il est à noter qu'une constance comparable doit être demandée 
pour les profondeurs et que par conséquent tout mouvement parasite du plan d'eau doit être proscrit (effet du vent par exemple);

b) Corrélativement si l'on se borne à étudier une suite discrète de valeurs de la fréquence, on pourra avoir la malchance de tomber à côté des fréquences les plus dangereuses ou encore de ne tomber que sur elles, ce qui, dans un cas comme dans l'autre, peut conduire à une interprétation erronée. Ceci suggère de faire les essais avec une onde incidente dont la fréquence varie d'une façon continue au cours du temps.

Si l'on suppose que la fréquence doit être définie avec une précision de l'ordre du millième pour que les résonances s'établissent sans ambiguïté et que le temps d'établissement de ces résonances est de l'ordre d'une minute (ce qui peut se vérifier sur modèle), on voit que la variation de fréquence devra être de l'ordre de 1/1.000 par minute. Par exemple, pour essayer des périodes variant de 1 a 3 , il faut environ 17 heures de mesure.
Il est bien clair d'ailleurs que cette variation de fréquence ne dispense pas de la précision car le programme établi doit être suivi avec beaucoup de continuité si l'on ne veut pas « manquer » certaines résonances par suite de sauts trop brusques.

On voit done apparaître l'exploitation d'un modèle de seiches comme une série d'enregistrements faits en principe à points fixes pendant que la fréquence glisse insensiblement d'une valeur limite à une autre. L'opération peut avoir à être répétée pour de nombreux niveaux de nlarée pour chaque aménagement étudié.

L'utilisation des résultats ainsi obtenus nécessite une connaissance ou au moins des hypothèses suffisamment précises sur la nature des ondes de seiches réelles (probabilités des fréquences et des largeurs de spectres). Il est à noter, d'ailleurs, que de telles connaissances ou hypothèses peuvent permettre d'étudier directement sur le modèle des seiches produites par des ondes irrégulières telles que celles auxquelles on peut s'attendre dans la nature. En l'absence de données statistiques sérieuses, cette façon de procéder ne nous semble pas recommandable.

\section{CONCLUSIONS}

Les problèmes de seiches dans les ports doivent s'étudier avec une optique particulière adaptée à l'échelle des longueurs d'onde de seiches.

Il est également essentiel de réaliser que l'extrême petitesse des cambrures des ondes de seiches leur confère un comportement très différent de celui des houles. En définitive, de toutes ces considérations, il se dégage que :

Les études préliminaires doivent se faire à l'échelle océanique, en principe au moyen d'épures de réfraction, les modèles ne pouvant tenir compte convenablement de la courbure lerrestre.

Le plateau continental et des structures à grande échelle analogues doivent parfois faire l'objet d'études spéciales. Dans certains cas, un modèle préliminaire représentant unè grande étendue à très petite échelle pourra être nécessaire.

L'étendue représentée par le modèle proprement dit doit être, en général, beaucoup plus vaste que pour une étude de houle. Ainsi l'échelle horizontale est-elle faible.
La distorsion des fonds devient précieuse du fait de la petitesse de l'échelle horizontale. Elle est heureusement autorisée dans une certaine mesure par les caractéristiques des ondes de seiches.

L'exagération des amplitudes nécessaire pour la précision des mesures, loin de fausser systématiquement la similitude des réflexions comme sur les modèles à houle, tend au contraire à corriger l'effet de distorsion. Cependant, il faut se garder d'exagérer les cambrures.

La réalisation de conditions aux limites convenables pose un des problèmes les plus importants de l'étude des seiches et conduit à donner aux amortisseurs et aux filtres un développement considérable.

Le réglage des caractéristiques de l'appareil générateur d'ondes pose des problèmes particulièrement délicats en ce qui concerne la constance de la fréquence qui doit pouvoir être réalisée avec une grande précision. L’étude en fréquence lentement variable semble s'imposer pour les problèmes de seiches étant donné que les conditions de résonance varient parfois rapidement en fonction de la fréquence. 
En terminant ces apercus, il nous semble utile d'insister à nouveau sur le fait que nous avons limité la discussion aux modèles concus en fonction d'une hypothèse particulière sur l'origine des seiches. Si d'autres hypothèses sont envisa- gées la conception des modèles pourra en être notablement modifiée; cependant la majorité des conclusions exposées ci-dessus garderont leur validité.

\section{BIBLIOGRAPHIE}

[1] BIEses. - The similitude of scale models for the study of seiches in harbours. Coastal Engineering, 1954, p. 95.

[2] Biesel, Suquet. - Les appareils générateurs de houle en laboratoire. La Honille Blanche, $\mathrm{n}^{\circ * 2}$, $4,5,1951 ; 6,1952$.

[3] Carlotri. - Contribution à l'étude de la houle au voisinage des côtes. La Houille Blanche, novembre-décembre 1947.

54] Greslov, Mahr. - Etude du coefficient de réflexion d'une houle sur un obstacle constitué par un plan ineliné. Coastal Engineering, 1954, p. 68.

[5] Iribarnen. --. Corrientes y oscillaciones de resacas en el interior de los Puertos. Publicado en la Revista de Obras Publicas, Madrid, avril 1948.
[6] LE Ḿ́hautš. - Two dimensional seiche in a basin subjected to incident waves. Coastal Engineering, 1954 , p. 119.

[7] McNows. - Sur l'entretien des oscillations des eaux portuaires sous l'action de la haute mer. Pablications Scientifiques et Techniques du Ministère de l'Air, no $278,1953$.

[8] MaNown, Danes. - Seiches in Harbours. The dock and harbor authority, $\mathrm{n}^{\circ} 384$, vol. XXXIII, p. 177 .

[9] Mrche. - Les mouvements ondulatoires de la mer. Annales des Ponts et Chanssées, 1944.

[10] Le Coin du Iaboratoire. La Houille Blanche, $\mathrm{n}^{\circ} 3$, 1955 .

\section{ERRATUM}

$\mathrm{N}^{\circ}$ 2. 1955, p. $134,1^{\text {re }}$ colonne :

Lire : $\varphi_{2}=-a \propto \frac{k}{m} \cdots$

et non : $\varphi_{2}=-a+\bar{a} \frac{k}{m} \cdots$ 